Man and Nature

L'homme et la nature

\title{
Les débuts de la Maçonnerie au Québec
}

\section{Jean-Paul de Lagrave}

Volume 7, 1988

URI : https://id.erudit.org/iderudit/1011936ar

DOI : https://doi.org/10.7202/1011936ar

Aller au sommaire du numéro

Éditeur(s)

Canadian Society for Eighteenth-Century Studies / Société canadienne d'étude du dix-huitième siècle

ISSN

0824-3298 (imprimé)

1927-8810 (numérique)

Découvrir la revue

Citer cet article

de Lagrave, J.-P. (1988). Les débuts de la Maçonnerie au Québec. Man and Nature / L'homme et la nature, 7, 195-207. https://doi.org/10.7202/1011936ar

Copyright (c) Canadian Society for Eighteenth-Century Studies / Sociéte canadienne d'étude du dix-huitième siècle, 1988
Ce document est protégé par la loi sur le droit d'auteur. L'utilisation des services d'Érudit (y compris la reproduction) est assujettie à sa politique d'utilisation que vous pouvez consulter en ligne.

https://apropos.erudit.org/fr/usagers/politique-dutilisation/ 


\section{Les débuts de la Maçonnerie au Québec}

Dans la ville de Québec, nouvellement conquise en 1759, le premier geste des officiers du général James Wolfe fut de fonder une grande loge provinciale c'est-à-dire d'établir un empire maçonnique dans le territoire acquis à la Grande-Bretagne. Mais il ne faut pas en déduire que la Franc-Maçonnerie n'existait pas auparavant sur les rives du SaintLaurent. Bien au contraire. Montréal est le siège d'au moins une loge de langue française composée de Canadiens, sans parler des loges que comportaient habituellement les régiments royaux de France.

Cet exposé donnera une esquisse de la présence de la Maçonnerie au Québec de 1759 à la fin du siècle. Il sera alternativement question des loges d'obédience française, puis anglaise. Des listes d'adhérents seront scrutées, de façon à retracer l'origine sociale des initiés. Il sera aussi traité de l'attitude des Maçons du Québec à l'occasion de la guerre d'Indépendance des États-Unis d'Amérique et de la Révolution française.

\section{Les Frères du Canada}

Bien que les bulles antimaçonniques de 1738 et de 1751 n'aient jamais été promulguées dans l'ex-Nouvelle-France, devenue la Province de Québec, le supérieur des Sulpiciens et seigneur de Montréal, Étienne Montgolfier s'élève contre l'Ordre en 1771, ce qui permet à l'historien de recueillir des données intéressantes dans la correspondance ecclésiastique. L'occasion en est le refus qu'un Franc-Maçon remplisse les fonctions de marguillier de Notre-Dame de Montréal, l'unique paroisse de la ville.

Voici la lettre la plus significative, datée du 20 janvier 1771, écrite par Montgolfier à l'évêque de Québec, Mgr Jean-Olivier Briand:

Nous avons un grand nombre de Francs-Maçons dans cette ville. Il y en avait quelques-uns, mais en petit nombre et cachés, sous le gouvernement français. 
Plusieurs de nos négociants ayant passé en France au temps de la révolution [c'est-à-dire au lendemain de la conquête] pour y arranger leurs affaires, s'y sont laissé séduire. La liberté du gouvernement présent leur laisse celle de se manifester; plusieurs ne craignent pas de le faire.

Il y a déjà plusieurs années que quelques-uns par surprise et incognito se sont insinués dans les assemblées des marguilliers; ils n'étaient pas ou peu connus sous le nom de leur société; ils ne fréquentaient pas les loges; personne n'en était scandalisé; j'ai cru qu'il était prudent de se taire et de les laisser passer.

Cependant c'est par une brigue de ses confrères cachés (je le sais) que le sieur [Pierre] Gamelin a été choisi en la dernière élection. Il était plus connu que bien d'autres, mais ayant promis qu'il ne tiendrait plus de loge, j'ai cru que ce serait une bonne occasion de le retirer comme ceux qui l'avaient précédé; et il a passé comme eux.

L'éclat de la cérémonie francmaçonne, dans laquelle il a paru publiquement le 3 de ce mois a un peu remué les esprits. Il m'eut été facile d'apaiser toutes choses en engageant ce monsieur, sans bruit et à l'amiable, à me donner sa démission de marguillier, car je ne pouvais pas espérer qu'étant maître de loge il y renoncerait absolument; et je sentais que plus on ferait de bruit, plus il se croirait obligé par honneur à soutenir ses démarches. Mais on m'a fait faire malgré moi une faute, mais qui heureusement n'a pas eu de mauvaise suite; c'est de l'avoir passé dans les visites que je rendais dans son quartier au commencement de l'année. Il y a été sensible, et cette circonstance a failli mettre obstacle à un accommodement qui cependant a été heureusement conclu dimanche dernier, 13 du courant. En voici les démarches et la conclusion.

Ce jour-là j'ai mandé honnêtement ce monsieur. Il m'est venu trouver avant la grand-messe. Je lui ai d'abord fait quelque excuse de l'avoir passé dans mes visites, en l'assurant que c'était contre mon inclination, mais seulement pour ménager la délicatesse d'un certain public ignorant. Il m’a avoué sa sensibilité, puis nous sommes entrés en matière.

Je lui ai fait lire la décision de la Sorbonne de 1745, et les bulles des souverains pontifes, ainsi que le tout se trouve dans l'abrégé du dictionnaire de Pontas, tome second, page 1382. Je lui ai fait sentir ce qu'il devait à la religion, à sa patrie, à sa famille, et ce qu'il se devait à lui-même, et l'incompatibilité des assemblées francmaçonnes avec celles des marguilliers, et l'ai prié d'opter entre les deux parties. Il comptait son honneur intéressé de part et d'autre, cependant il m'a promis qu'il renoncerait aux loges. Je ne me repose qu'à demi, ou même pas du tout sur cette promesse. Mais j'espère bien que s'il y paraît, ce ne sera que rarement, très secrètement et sans scandale, et que la faute lui sera purement personnelle. Nous nous sommes quittés sur cela et paraissant contents l'un de l'autre. 
Après cette première démarche, j'ai fait convoquer une assemblée de marguilliers pour le même jour. On ne savait pas où en était cette affaire et on s'attendait qu'elle serait mise sur le tapis. L'assemblée n'a pas été aussi nombreuse que je l'aurais désiré; mais cependant il s'y est trouvé quelques Francs-Maçons et autres.

Je leur ai fait faire la même lecture que j'avais faite le matin au sieur Gamelin, puis partant de ce principe, je leur ai représenté que de tous temps l'assemblée des marguilliers n'avait pas été seulement une assemblée d'honnêtes gens selon le monde, mais de chrétiens fervents et soumis à l'Église; qu'ils savaient ce qu'avaient été leurs ancêtres et qu'ils ne devaient pas souffrir qu'elle dégénérât, et fût composée d'enfants rebelles à l'Église et excommuniés; que je les en laissais eux-mêmes les juges; que je n'étais pas surpris que plusieurs jeunes gens séduits et curieux eussent pris parti dans la société francmaçonne; mais je le serais grandement si des gens graves et des pères de famille demeuraient attachés à des assemblées qui, quand elles ne seraient pas criminelles et impies, seraient au moins puériles et indignes d'eux; que je ne voulais pas toucher à ceux de cette société qui avaient été choisis jusqu'à présent marguilliers, soit qu'on les eût connus auparavant, ou non; que j'espérais qu'ils se retireraient d'eux-mêmes de l'une ou l'autre assemblée; mais que pour l'avenir dans l'élection des marguilliers, on supprimerait entièrement les noms de tous ceux qui seraient soupçonnés d'être Francs-Maçons, à moins qu'il ne donnassent des marques suffisantes qu'ils y avaient renoncé. Tous ces messieurs ont paru entrer dans ces vues, et ont rapporté dans leur famille l'idée qu'on devait avoir chrétiennement des Francs-Maçons. ${ }^{1}$

Cette lettre de Montgolfier nous apprend qu'en 1771 il existait à Montréal un important groupe de Maçons de langue française, dont plusieurs avaient été initiés en France vers 1760-1763. Beaucoup de jeunes gens étaient attirés par l'esprit maçonnique, de même que les hommes les plus graves. Des cérémonies maçonniques avaient même lieu publiquement. Dans cette même lettre Montgolfier confirme le fait qu'il y avait une Maçonnerie en Nouvelle-France. Qu'après la conquête, des loges francophones continuèrent à fonctionner.

Pourquoi s'élever contre l'Ordre précisément en 1771? Cette annéelà les Franc-Maçons de langue française s'imposent même dans les loges britanniques. Installée le 17 juin 1769, la St. Peter no 4 décidait le 24 juin 1771 de faire ses travaux en français à l'une de ses deux tenues mensuelles. ${ }^{2}$ Le négociant Pierre Gamelin, dont parle Montgolfier dans sa lettre, y était alors deuxième surveillant. Le maître était Jean Rochat et le premier surveillant, François Picotté de Bélestre, chevalier de SaintLouis. Parmi les autres membres de la loge St. Peter, nous pouvons citer l'avocat Michel-Amable Berthelot d'Artigny, Jean-Baptiste Céloron 
de Blainville, Benjamin-Mathieu d'Amours de Cligancourt, Charles Curot, Jean Dumoulin, Louis Ermatinger, Jean-Baptiste-Melchior Hertel de Rouville, Joseph-Guillaume de Lorimier, l'avocat Simon Sanguinet, Louis Verchères. ${ }^{3}$ Cette loge regroupait les notables de langue française à tendance extrêmement loyaliste, provenant en partie des familles nobles ou possédant des seigneuries. Des mariages renforçaient les liens familiaux et maçonniques. Ainsi Benjamin-Mathieu d'Amours de Cligancourt épousa Catherine de Lorimier et Joseph-Guillaume de Lorimier Maria Madeleine d'Amours de Cligancourt. ${ }^{4}$

Une autre loge fut constituée de Maçons à tendance libérale; elle fut composée en grande partie de bourgeois. Ce fut celle des Frères du Canada, instituée en $1785 .{ }^{5}$ Elle était formée de notables qui gravitaient autour du premier imprimeur-libraire de Montréal, Fleury Mesplet. Le maître en était le notaire Jean-Guillaume Delisle de la Cailleterie, qui avait été délégué à Londres en 1783 pour réclamer entre autres une chambre d'assemblée. ${ }^{6}$ Le gardien du sceau était Jacques-Clément Herse, ouvrier-imprimeur venu de Philadelphie avec Mesplet et devenu négociant. ${ }^{7}$ Louis l'Hardy, qui s'intitule dans quelques documents 'ami' de Mesplet, était le 'fournisseur' de la loge. ${ }^{8}$ Au nombre des autres Frères du Canada, nous trouvons les organistes Louis Champagne 9 et Antoine Tabeau, de la famille propriétaire du second atelier de l'imprimeur; ${ }^{10}$ Philippe de Rocheblave, collaborateur à la Gazette de Montréal; ${ }^{11}$ Pierre Marassé, qui aida Mesplet dans ses revendications auprès du Congrès des États-Unis, de même que Étienne Fournier et Alexander Henry. ${ }^{12}$

Bien que Mesplet n'apparaisse pas au tableau des Frères du Canada qui nous est connu, celui de 1788 , ni sur une attestation notariale de 1790 donnant les noms des dirigeants, ${ }^{13}$ le portrait qui nous est parvenu de lui ${ }^{14}$ est attribué au peintre François Malepart de Beaucourt, qui s'identifie comme Franc-Maçon. L'imprimeur est représenté debout au comptoir de sa librairie avec à sa droite la colonne B., évocation dans la symbolique maçonnique de la puissance du travail persévérant que nul obstacle ne rebute. ${ }^{15}$ Dans quelques unes de ses toiles, la signature de Beaucourt est suivie des trois points posés en triangle. ${ }^{16}$ Herse, le garde du sceau des Frères du Canada, signe de la même façon dans l'attestation notariale de 1790 , près du sceau en cire rouge représentant sur un écu l'oeil du Grand Architecte de l'Univers, deux mains fraternellement unies au-dessus de l'adjectif Inséparable, et des feuilles d'acacia. ${ }^{17}$ À noter que l'année de fondation de cette loge fut aussi celle de la naissance de la Gazette de Montréal, le grand périodique d'information de Mesplet. ${ }^{18}$

Un autre atelier des Frères du Canada existait à Québec. Un document, qui est un compte rendu d'une dernière tenue, ${ }^{19}$ atteste le lien 
avec la loge montréalaise et donne une liste d'initiés à savoir, les avocats Pierre-Louis Panet, un anti-esclavagiste, ${ }^{20}$ Antoine Méru Panet, Bonaventure Panet; l'imprimeur P.-E. Desbarats, ${ }^{21}$ Pierre Labadie, le notaire Pierre Grisé, le linguiste François Gamelin Launière, Gaspard Tarieu de Lanaudière. ${ }^{22}$ Le 8 juin 1788, les Frères du Canada de Québec se mettaient en sommeil et retournaient leur charte aux Frères du Canada de Montréal. ${ }^{23}$

Les Frères du Canada avaient pris la relève des Francs-Maçons régénérés ${ }^{24}$ qui avaient eu comme marraine la loge Amitié et Fraternité de Dunkerque fondée en $1743 .{ }^{25}$ Les Frères du Canada relevaient du Grand Orient de France. ${ }^{26}$ Ils obtinrent toutefois en 1816 à Québec une charte de la Grande Loge du Québec, signée par le grand-maître. ${ }^{27}$ Ce Perrault avait collaboré avec Mesplet dans la traduction du code des juges de paix. ${ }^{28}$ Les trois officiers nommés dans la nouvelle charte, y compris Perrault, étaient 'vénérables. ${ }^{29}$

La chanson des Frères du Canada exprime bien le message maçonnique vécu dans la Province de Québec dans la seconde partie du XVIIIe siècle. En voici le refrain et les sept couplets:

Vivons, aimons, chérissons la Concorde,

Chantons l'amour qui nous a réunis.

Dans nos plaisirs évitons la discorde

Soyons toujours d'un seul et même avis.

1

Par des égards, que chacun se prévienne, Soyons polis, complaisants sans fadeur. S'il se glissait entre nous quelque haine,

De la chasser, engageons notre honneur.

\section{2}

Que la vertu jamais de nous s'écarte Enchaînons-la dans un juste milieu. Nourrissons-nous d'une joie délicate Qu'aucun excès n'avilisse nos jeux.

3

Point de pédant, maudissons cette race

Redoutons-la, autant que le poison.

Elle décide toujours avec audace,

Et bien souvent, sans rime ni raison. 
Dans nos plaisirs qu'aucune inquiétude

Ne porte obstacle à nos amusements.

Ayons pour nous cette aimable habitude

De n'afficher que le contentement.

5

Par des bienfaits, signalons notre gloire, Soyons vertueux, à la mort, à la vie!

Que tous nos noms, au temple de mémoire,

À l'univers puissent porter envie.

\section{6}

Que nos promesses ne soient point chimère

Appliquons-nous tous à les maintenir.

Que notre amour soit ardent et sincère

N'en oublions jamais le souvenir.

\section{7}

Sur les débris du plus grand des naufrages,

Dans le néant, dit-on, tout tombera,

Consolons-nous en attendant l'orage

Et dans le temps se sauve qui pourra. ${ }^{30}$

Cette chanson définit un nouvel art de vivre dont les caractéristiques sont, par ordre d'insertion dans la pièce, la fraternité, l'égalité, la tolérance, la vertu, la liberté, la bienfaisance, le respect de la Société des Francs-Maçons et de ses règles. Le refrain insiste sur l'esprit fraternel qui doit animer les travaux des Frères du Canada: la Concorde prend la figure d'une égérie qu'il faut chérir. Le premier couplet invite les initiés à vivre en égaux, sans permettre à la haine de briser l'harmonie basée sur une exquise politesse. Le deuxième couplet rappelle cette pensée d'Horace: 'En toutes choses, il y a une juste mesure; il existe des limites fixes, au-delà ou en deça desquelles le bien ne saurait exister. ${ }^{\prime 31}$ Le troisième couplet est le seul qui marque une opposition à l'égard d'un groupe social, les 'pédants.' Leur fanatisme les rend redoutables, car ils ne font pas appel à la raison. Dans le quatrième couplet, l'auteur dit que les activités maçonniques doivent se dérouler sous le signe de la liberté dans la plus grande sérénité. Que la bienfaisance soit le signe de l'amour de l'humanité, soutient-on dans le cinquième couplet. Il est question d'une loyauté indéfectible envers les constitu- 
tions de l'Ordre dans le sixième couplet. Enfin, apparait dans le dernier couplet un autre rappel de la pensée d'Horace: 'Nous sommes tous poussés au même but; dans l'urne, notre sort à tous est agité, il sortira un peu plus tôt, un peu plus tard; mais nous tous prendrons passage dans la barque pour l'exil éternel. ${ }^{\prime 32}$ En bref, la chanson conseille le juste milieu en toutes choses. Le Maçon doit être un homme d'honneur, vertueux, serein, fidèle à ses promesses et n'attendant aucune autre récompense que la satisfaction de faire le bien.

\section{Les loges britanniques}

À part les loges des Frères du Canada, entre 1759 et 1791 la Province de Québec a compté 37 loges dont sept à Montréal. ${ }^{33}$ Après la prise de Québec, le lieutenant John Price Guinnett avait été proclamé grandmaître le 28 novembre 1759 , alors qu'avait été fondée une grande loge provinciale qui groupait six loges des régiments de Wolfe..$^{34}$ La Grande Loge d'Angleterre ne reconnut jamais l'élection de Guinnett, la considérant irrégulière. ${ }^{35} \mathrm{La}$ situation fut régularisée lors de l'installation du colonel Simon Fraser comme grand-maitre le 24 juin $1760 .^{36}$

En 1762, il y avait dans la province treize loges militaires et une de marchands à Québec, relevant toutes de la Grande Loge du Québec; ;7 en 1767, Edward Antill était nommé représentant du grand-maître de la loge provinciale à Montréal. ${ }^{38}$ La première loge britannique civile, St. Peter, était installée à Montréal le 17 juin $1769 .{ }^{39}$ Un an plus tard, une deuxième loge, St. Paul, était créée, ${ }^{40}$ et une troisième, Select, en 1782. ${ }^{41}$ Sous la grande-maîtrise de sir John Johnson le siège de la Maçonnerie fut transféré de Québec à Montréal en 1788: Thomas McCord, un membre de la loge St. Peter, fut nommé grand secrétaire et William Grant, représentant du grand-maître à Québec. ${ }^{42}$

La grande-maîtrise fut accordée au prince Edward, duc de Kent, le 22 juin 1792 par les deux grandes loges d'Angleterre. Depuis 1751, en effet, la Maçonnerie anglaise était divisée entre les Modernes et les Anciens, scission qui ne devait se résorber qu'en $1813 .{ }^{43} \mathrm{Au}$ moment de l'arrivée du duc au Canada il n'y avait que trois loges des Anciens au Québec. Il en créa sept en 1792, trois de plus en 1793 et deux en 1794. ${ }^{44}$ Au départ du prince en janvier 1794, une adresse lui fut présentée par William Grant, le député grand-maître des Modernes et par Thomas Ainslie, le député grand-maître des Anciens. ${ }^{45}$

Les Maçons se manifestaient publiquement dans la province. Ainsi en 1787, à Québec, l'hôtel du Chien d'or, devenu le Freemasons' Hall, fut solennellement dédié à la Maçonnerie le 3 novembre, en présence du gouverneur général Guy Carleton, lord Dorchester, de lady Maria 
Dorchester et de nombreux invités de marque. ${ }^{46}$ Le pasteur Alexander Spark, qui prendra éventuellement la direction de la Gazette de Québec, prononça l'homélie de circonstance. ${ }^{47}$ Ce même journal, dans son numéro du 28 mars 1793, donne la description des funérailles maçonniques du boulanger Alexander Galloway dont le corps 'fut accompagnée d'une manière très solennelle par son Altesse Royale le prince Edouard ... et par tous ses frères alors sous sa juridiction en cette ville.' Le corps était 'sur un chariot, accompagné de six porteurs du drap mortuaire, avec des bandolières blanches et des bandes blanches autour de leurs chapeaux. Toute la loge 241 en deuil, avec des bandes blanches autour de leurs chapeaux, et des gants blancs, portant chacun une branche verte. ${ }^{\prime} 48$

Les Maçons ne négligeaient pas la publicité dans la presse. Ainsi dans la Gazette de Québec du 21 juin 1764, un appel d'adhésion à la Merchants' Lodge no 1 est lancé. ${ }^{49}$ Cette loge, composée de commerçants, avait été fondée au début de $1760 .{ }^{50}$ Dans la Gazette de Montréal, Mesplet traite de la Franc-Maçonnerie surtout à l'occasion des Saint-Jean d'hiver et d'été en publiant des convocations ou des allocutions de circonstance. ${ }^{51}$ Ainsi, il donne un discours du secrétaire de la loge l'Union d'Albany, prononcé le 24 juin 1785, dont voici un extrait exprimant le voeu d'unir les Maçons des nouveaux États-Unis d'Amérique et ceux du Canada, en oubliant le récent conflit avec la Grande-Bretagne:

Si dans le cours de la dernière révolution de l'Amérique il a été fait quelques brèches à nos murs, si la chaîne d'or qui unit toutes les parties de la Fraternité à contracté quelques rouilles, ou si les cordes de notre tabernacle ont été desserrées, comme cela regarde particulièrement la dignité de notre institution, et votre honneur, je vous conjure par les devoirs les plus sacrés de réparer immédiatement ces brèches, de dérouiller et polir cette chaîne, de resserrer ces cordes de manière qu'elles durent éternellement. ${ }^{52}$

Les annonces maçonniques que publie Mesplet, sauf celles des Frères du Canada, ${ }^{53}$ sont uniquement en anglais et sont officiellement signées, par exemple le 5 juin 1788, par le grand trésorier de la Grande Loge du Québec, John Gerbrand Beck. ${ }^{54}$

Voici quelques renseignements sur certains notables faisant partie des loges britanniques. Sir John Johnson, qui fut grand-maître, avait organisé un régiment loyaliste durant la guerre d'Indépendance des colonies américaines. De 1791 à sa mort, il fut superintendant des Affaires indiennes au Canada. Johnson avait été grand-maître provincial de New York en $1767 .{ }^{55}$ De Johnson, les historiens maçonniques américains ont écrit: 'ses yeux étaient devenus aveugles aux signes maçonniques et ses oreilles sourdes au mot du Maçon. ${ }^{\prime 56}$ Edward-William 
Gray fut initié à l'âge de 18 ans, à Québec en 1760 . Il obtint une commission de notaire à Montréal en 1765 . Il fut nommé directeur des postes dans cette même ville en 1778 . Il était un membre actif de la loge St. Peter. ${ }^{57}$ Au cours d'une vente publique, Gray acheta en 1785 les presses de Mesplet et les lui 'prêta' ensuite à vie. ${ }^{58}$ William Moore, l'imprimeur du Quebec Herald, s'affichait comme Franc-Maçon et James Tanswell, l'éditeur du Héraut français, 59 était grand secrétaire de la Grande Loge du Québec en $1784 .{ }^{60}$ Adam Lymburner et William Grant, qui luttèrent pour une chambre d'assemblée, étaient aussi Maçons. ${ }^{61}$ Daniel Clause, de la direction des Affaires indiennes, qui accorda un important contrat d'impression à Marie Mirabeau-Mesplet durant l'emprisonnement de son époux, avait été initié à New York et était le beaufrère du grand-maître de la Grande Loge du Québec, sir John Johnson. ${ }^{62}$

Le maçon le plus célèbre à se manifester au Québec dans le dernier quart du XVIIIe siècle fut Benjamin Franklin, qui vint à Montréal en 1776, à titre de commissaire du Congrès américain. ${ }^{63} \mathrm{C}^{\prime}$ est son intervention qui dota Montréal de son premier imprimeur. ${ }^{64}$ Celui-ci, Fleury Mesplet, fut le diffuseur au Québec des idées philosophiques, ${ }^{65} \mathrm{dont}$ le plus éminent représentant, Voltaire, avait été initié à la loge des NeufSoeurs, à Paris, en présence de Franklin. ${ }^{66}$

On peut dire que les deux grands événements mondiaux qu'ont été la guerre d'Indépendance des États-Unis d'Amérique et la Révolution française ont déchiré la Maçonnerie au Québec, comme partout en Occident. Il y avait des Maçons parmi les Loyalistes, tel ce Johnson, déjà cité, devenu grand-maître des loges britanniques dans la province. Par contre, les leaders du mouvement de libération des colonies étaient Maçons, tels Washington, Franklin et LaFayette. ${ }^{67}$ Ce trio a sûrement influencé l'attitude de ses frères maçons parmi les négociants montréalais qui ont appuyé les Fils de la Liberté. ${ }^{68}$ La première Lettre du Congrès adressée aux habitants du Québec en 1774 est empreinte de l'idéal maçonnique, insistant sur la liberté, l'égalité et la fraternité et citant même un 'frère,' Montesquieu, pour les inciter à secouer le joug de l'Angleterre. ${ }^{69}$ Le même esprit se reflète dans l'appel des Français libres à leurs frères $d u$ Canada, adressé à la population par EdmondCharles Genet, ambassadeur de la République françaises à Philadelphie, en $1793 .{ }^{70}$ Le texte est un hymne à la Liberté. Cet appel ressemble à ceux que lançait, au nom de la France républicaine, Condorcet, membre comme l'avait été Voltaire, de la célèbre loge des NeufSoeurs. ${ }^{71}$ La Lettre de Genet aux Canadiens est le développement de l'article premier de la Déclaration des droits rédigée par Condorcet, article qui se lit comme suit: 'Les droits naturels, civils et politiques des hommes sont: la liberté, l'égalité, la sûreté, la propriété, la garantie sociale et la résistance à l'oppression. '72 'Tout autour de vous, vous 
invite à la liberté, écrit Genet aux Canadiens. Le pays que vous habitez a été conquis par vos pères. Il ne doit sa prospérité qu'à leurs soins et aux vôtres. Cette terre vous appartient. Elle doit être indépendante. ${ }^{73}$ À cette déclaration était joint l'ouvrage Les Droits de l'homme de Thomas Paine, Franc-Maçon, ami de Franklin et de Condorcet, ${ }^{74}$ ouvrage valorisant les idéaux de la Révolution française. Mesplet se chargea de faire circuler l'appel des Français libres aux Canadiens ainsi que le livre de Paine à Montréal et dans la province. ${ }^{75}$

En conclusion, disons que la conquête britannique a accéléré le rayonnement de la Franc-Maçonnerie au Québec. Car l'implantation de l'Ordre avait précédé la cession. Toutefois, selon l'affirmation même d'un opposant, le vicaire général Étienne Montgolfier, la Maçonnerie n'a vraiment commencé à s'extérioriser que sous le régime britannique. Alors, durant une quarantaine d'années, ont coexisté des loges d'obédience française et britannique. Dans celles-ci, la présence canadienne s'est manifestée et très tôt, assez pour que les hauts dignitaires de $l^{\prime}$ Eglise catholique s'en inquiètent. Les loges britanniques recevaient surtout des nobles et des seigneurs alliés, tandis que les loges françaises étaient remplies d'éminents membres de la bourgeoisie. Les bourgeois d'origine écossaise se répartissaient dans les deux obédiences; les anciens militaires demeuraient fidèles aux loges britanniques. Les listes de Francs-Maçons qui nous sont parvenues montrent à l'évidence que les initiés comptaient parmi les notables de la colonie et qu'ils occupaient des fonctions capables d'influencer l'opinion. L'augmentation rapide du nombre des loges britanniques a finalement donné à la Maçonnerie du Québec un cadre conservateur, favorable au maintien du lien avec la Grande-Bretagne, ce qui n'a toutefois pas empêché l'expression d'une Maçonnerie d'esprit libéral: plusieurs Maçons ont été réformistes dans le dernier quart du XVIIIe siècle au Québec.

\author{
JEAN-PAUL DE LAGRAVE \\ Montréal
}

\title{
Notes
}

1 Lettre d'Étienne Montgolfier à l'évêque de Québec datée du 20 janvier 1771: Archive de la chancellerie de l'archidiocèse de Montréal, correspondance de Montgolfier (1771-1775), 901-1005, 771. 
2 'When the nobility and aristocracy of French Canada favored Free Masonry': exposé donné au Masonic Study Club de Westmount par C.E. Holmes, Fonds Patenaude, Université du Québec à Montréal, p. 17. Voir aussi A.J.B. Milborne, Freemasonry in the Province of Quebec 1759-1959 (Knowlton: Milborne, 1959), p. 22.

3 'Nobility and Free Masonry,' pp. 17-19.

4 Ibid., p. 18.

5 Charles E. Holmes, 'Les Frères du Canada - The Brethren of Canada,' Masonic Light (octobre, 1947), pp. 44, 45.

6 Jean-Paul de Lagrave, Fleury Mesplet (1734-1794), diffuseur des Lumières au Québec (Montréal: Patenaude Éditeur, 1985), pp. 259, 494.

7 Mesplet, pp. 209, 494. Voir aussi 'The Nobility and Free Masonry,' p. 11.

8 Mesplet, pp. 209, 253, 256, 494.

9 'The Nobility and Free Masonry,' p. 12.

10 Mesplet, p. 75. Voir aussi Les Frères du Canada, p. 41.

11 Mesplet, pp. 267, 494.

12 Ibid., pp. 202, 208, 494, 495.

13 Les Frères du Canada, pp. 40, 44.

14 Mesplet, pp. 439-42.

15 Edouard E. Plantagenet, Causeries initiatiques pour le travail en loge d'apprentis (Paris: Dervy, 1962), p. 126.

16 Madeleine Major-Fregeau, La vie et l'oeuvre de François Malepart de Beaucourt (Québec: Ministère des Affaires culturelles, 1979), p. 40.

17 Archives nationales du Québec à Montréal: Lettres et documents avec sceaux, 1776-1906, vol. 2: $06 \mathrm{M} \mathrm{CD} 1$ - 1/2.

18 Mesplet, p. 217.

19 Les Frères du Canada, p. 48.

20 'The Nobility and Free Masonry,' pp. 9-10.

21 lbid., p. 9.

22 Ibid., p. 9, 10.

23 Ibid., p. 11.

24 Charles H. Holmes, 'French Canadian Masons of Yesterday,' conférence prononcée à l'occasion du $75 \mathrm{e}$ anniversaire de la loge des Coeurs unis, en 1945, pp. 8, 9.

25 F. Weil, 'La Franc-Maçonnerie en France jusqu'en 1755,' Studies on Voltaire and the Eighteenth Century, vol XXVII (1963), 1812.

26 Les Frères du Canada, p. 48.

27 'The Nobility and Free Masonry,' p. 4. Lire 1816 et non 1792.

28 Mesplet, p. 296.

29 'The Nobility and Free Masonry,' p. 4. 
30 E.Z. Massicote, 'La chanson des Frères du Canada,' Bulletin des recherches historiques, vol XXVI (mai 1920), 152-53.

31 Horace, Odes, Chant séculaire, épodes, satires, épîtres, Art poétique (Paris: GarnierFlammarion, 1967) pp. 149-150 (Satire 1, livre premier).

32 Ibid., 73 (Ode 111, livre 11).

33 H. Graham, Outlines of the History of Freemasonry in the Province of Quebec (Montréal: Lovell, 1892) pp. 38-39.

34 Milborne, Freemasonry in Quebec, pp. 2, 3.

35 Ibid., p. 6.

36 Ibid., p. 8.

37 Ibid., p. 13.

38 Ibid., p. 15.

39 Ibid., p. 22.

40 Ibid., p. 24.

41 Ibid., p. 32.

42 Ibid., p. 40.

43 Paul Naudon, Histoire générale de la Franc-Maçonnerie (Paris: Presses universitaires de France, 1981), pp. 40-42.

44 Milborne, Freemasonry in Quebec, p. 47.

45 Ibid., p. 46.

46 Ibid., pp. 4, 5.

47 Ibid. Voir Mesplet, p. 397.

48 Gazette de Québec, 28 mars 1793.

49 Gazette de Québec, 21 juin 1764.

50 Graham, History of Freemasonry, p. 41.

51 Par exemple, les numéros des 26 janvier 1786, 11 mai 1786, 13 septembre 1787, 18 septembre 1788 .

52 Gazette de Montréal, 26 janvier 1786.

53 Exemples de textes sur les Frères du Canada dans les numéros des 13 septembre 1787 et 18 septembre 1788 .

54 Gazette de Montréal, 5 juin 1788.

55 Milborne, Freemasonry in Quebec, p. 38.

56 Ibid., p. 40.

57 Ibid., p. 87.

58 Mesplet, p. 222.

59 Ibid., p. 446.

60 Ibid.

61 Ibid. 
62 Ibid.

63 Ibid., pp. 63, 72.

64 Ibid., p. 65.

65 Ibid., p. 1 (introduction).

66 Ibid., p. 447.

67 Jean-André Faucher, Dictionnaire maçonnique (Paris: Jean Picollec, 1981) pp. 111, 162, 290.

68 Mesplet, p. 35.

69 Ibid., pp. 26-30.

70 Ibid., pp. 409-12.

71 Frank Alengry, Condorcet, guide de la Révolution française (New York: Burt Franklin, 1973), p. 19.

72 lbid., p. 371.

73 Mesplet, p. 410.

$74 \mathrm{H}$. Archambault de Montfort, Les idées de Condorcet sur le suffrage (Genève: Slatkine Reprints, 1970), p. 134.

75 Mesplet, pp. 414, 419. 